\title{
El proceso de evaluación del aprendizaje antes y durante la contingencia sanitaria por Covid-19. Un estudio exploratorio en secundarias públicas mexicanas.
}

\author{
The evaluation process of learning before and during Covid-19 lockdown. An \\ exploratory study in Mexican public secondary schools.
}

\author{
Rosa A. Espinoza-Cid ${ }^{a}$, Isis B. Caro-Coronado ${ }^{b}$
}

\begin{abstract}
:
In March 2020, México declared a health emergency due to the increase of confirmed cases of Covid-19. Because of the associated risks, all government levels cancelled in-school classes for all educational institutions in the country, switching into a distance-based model for education. With this new work model, the assessment dynamics of learning have changed. Besides this, in 2017, the Secretariat of Public Education (the education office in México) implemented a transformative change in the assessment procedures. Particularly, concerning to the periods for evaluating the students' learning during the scholar period; and the adaptations that would depend on the teachers' reflection on the results in order to improve their teaching practice and the educational process. For both situations, it is important to know the teachers' perceptions, so that if necessary, they can take any steps and modify the teachers' assessment perceptions accordingly to the results. This investigation is exploratory and has the main objective of having a first view to the definition of techniques, ways, and criteria for assessing students learning in the English class in secondary schools in a city located in the north-west of Mexico. The results showed that participants have similar perceptions of their definitions and criteria for assessment, and they coincide that throughout the pandemic contingency, they have faced different changes in their perceptions about the evaluation process.
\end{abstract}

Keywords:

Assessment, distance education, English (second language), secondary school.

\section{Resumen:}

En marzo de 2020 México decretó la emergencia sanitaria por múltiples casos confirmados de Covid-19. Debido a los riesgos implicados, todos los niveles de gobierno, declararon la suspensión de clases presenciales en las escuelas del país, transitando hacia un modelo de educación a distancia. Con esta nueva modalidad de trabajo, las dinámicas tradicionales de evaluación de los aprendizajes se han visto modificadas. A manera de antecedente, en 2017, la Secretaría de Educación Pública implementó la Reforma a la educación básica, la cual implicó diversos cambios al proceso de evaluación; particularmente a los periodos contemplados para la evaluación durante el ciclo escolar, así como adaptaciones dependientes de la reflexión de los profesores sobre los resultados para mejorar su práctica docente y el proceso educativo. A partir de ambos acontecimientos, resultó importante conocer las percepciones de los docentes, para que, de ser necesario, se tomen medidas y realicen las modificaciones pertinentes. Esta investigación, de tipo exploratorio, tuvo como objetivo realizar un acercamiento a definiciones, técnicas, formas y criterios para evaluar el aprendizaje en la asignatura de inglés de escuelas secundarias públicas, desde la perspectiva de los profesores, además de conocer el tránsito del proceso evaluativo de los alumnos en el modelo educativo a distancia. Fueron entrevistados ocho maestros de inglés de secundaria en una ciudad del noroeste de México. El análisis indicó que los participantes coinciden en sus definiciones y criterios de evaluación y que, durante la contingencia han enfrentado diversos cambios en sus percepciones sobre el proceso de evaluación del aprendizaje.

\section{Palabras Clave:}

Evaluación, educación a distancia, educación secundaria, inglés (segunda lengua).

\footnotetext{
Rosa A. Espinoza-Cid, Escuela Normal Superior, plantel Hermosillo, https://orcid.org/0000-0003-1327-5364, Email: ensh.respinoza@creson.edu.mx

$b$ Isis B. Caro-Coronado Escuela Normal Superior, plantel Hermosillo, https://orcid.org/0000-0002-4513-1827, Email: ensh.icaro@creson.edu.mx
} 


\section{INTRODUCCIÓN}

En un hecho sin precedentes en el mundo moderno, durante finales del 2019 y comienzos del 2020, los gobiernos comenzaron a decretar medidas en un intento de contrarrestar la propagación de la Covid-19. En México, una de las principales medidas fue el cierre de los planteles de todos los niveles educativos. De acuerdo a estadísticas de educación básica de la publicación Principales Cifras del Sistema Educativo Nacional 2019-2020 (SEP, 2020), 6, 307, 056 alumnos en secundaria en el país, se vieron afectados tras el cierre de todos los planteles de educación el 20 de marzo de 2020, emigrando a modalidad a distancia, las clases que siempre habían sido en modalidad presencial.

Con motivo de frenar la propagación de la Covid-19, y anticipar los próximos desafíos por emergencia sanitaria mundial, la UNESCO (2020) emitió el documento Respuesta del ámbito educativo a la Covid-19: preparación para la reapertura de las escuelas. El objetivo de éste es hacer saber a las autoridades educativas sobre las consideraciones a tomar, decretar las políticas públicas conforme a los contextos de cada región para el momento de la reapertura, así como dar cuenta de lo que han hecho otros países en situaciones similares. Igualmente, se reconoce que el sistema educativo debe de ser eficaz en distintos puntos; específicamente para la evaluación, se centra en que, tanto autoridades del plantel, como docentes, se preparen pedagógicamente para determinar qué se debe de evaluar, y así poder continuar evaluando el progreso y aprendizaje del estudiantado durante tiempo que las escuelas permanezcan cerradas. Además de lo anterior, evaluar la pérdida del aprendizaje provocado por el periodo de aislamiento, con el fin de organizar cursos de recuperación, y enfocarse en los objetivos más importantes al momento de diseñar exámenes (UNESCO, 2020).

Anterior a la pandemia de Covid-19, en agosto de 2017, el ciclo escolar en educación básica, comenzó de la mano con un nuevo modelo educativo para todo el país. Esta reforma, implementada por la Secretaría de Educación Pública (SEP), articula la educación obligatoria, la cual contempla desde educación inicial, hasta educación media superior, e incluye también el rediseño de los planes de estudio de las escuelas Normales de todo el país. En educación secundaria, particularmente en la asignatura de lengua extranjera: inglés, los efectos de reformas anteriores han mostrado resultados deficientes. En el último reporte del Índice de Competencia en Inglés (EPI-s, 2019), señala que México se encuentra en el lugar 67 de 100 países participantes, con un nivel bajo de aptitud en la lengua. Además, en reportes de organizaciones financiadas con recursos privados arrojaron como resultado en inglés de los alumnos que terminan secundaria que no saben inglés, señalando que no cuentan con los conocimientos esenciales para acreditar el primer nivel del MCER A1 (Mexicanos Primero, 2015), cuando según el actual plan y programas de estudio, al finalizar este nivel educativo, el alumnado alcanzaría un nivel B1, tras haber recibido un total de 1060 horas de instrucción recibidas desde tercero de preescolar hasta tercero de secundaria (SEP, 2017).

Por lo anterior, es importante continuar investigando sobre los procesos de enseñanza aprendizaje del inglés en el sistema educativo de nivel básico público mexicano. En el caso de la presente investigación, se enfoca en varios aspectos del proceso de evaluación de los aprendizajes en la clase de inglés secundaria, desde la perspectiva del docente de inglés. De acuerdo al documento Aprendizajes Clave para la Educación Integral (SEP, 2017) el profesor es el encargado de (re)diseñar el proceso de evaluación continuo, permanente y formativo, a través de la selección de instrumentos, criterios y estrategias adecuadas al alumno que le faciliten el proceso de valoración del aprendizaje del mismo. En la enseñanza del inglés esto resulta primordial debido a que, la evaluación, en cualquiera de sus formas, por ejemplo, a través de un examen escrito, es también una forma en que los estudiantes ganan nuevos conocimientos (Tomilson, 2005). Además, de acuerdo a Gultom (2016) en la asignatura de inglés la evaluación se sugiere debe ser continua a través de la observación constante del trabajo de los alumnos, así como del registro en cada sesión de las participaciones de estos.

\section{LA EVALUACIÓN EN EL PLAN Y PROGRAMAS DE ESTUDIO DE EDUCACIÓN BÁSICA}

La perspectiva de la SEP con respecto a los procesos de evaluación en educación básica es la de mejorar la calidad de los procesos de enseñanza y de aprendizaje. La SEP propone dentro del panorama del documento Aprendizajes Clave para la Educación Integral y los principios pedagógicos con la finalidad de que "el docente consiga transformar su práctica y cumpla plenamente su papel en el proceso educativo al poner en marcha los objetivos" (2017, p. 118). Estos a su vez incluyen cuatro variables de evaluación "en relación con la planeación del aprendizaje” (SEP, 2017, p. 120): las situaciones didácticas, las actividades del estudiante, los contenidos y la reflexión del docente sobre su práctica.

Conforme a Aprendizajes Clave, las evaluaciones y su comunicación a los padres de familia y/o tutores está calendarizada en tres periodos denominados trimestres que van de agosto a noviembre, de diciembre a marzo y de abril a julio. Dentro de estos periodos trimestrales se realizan evaluaciones generales y específicas. Estas últimas corresponden a los aprendizajes esperados. Lo anterior se somete a adaptaciones por parte del profesorado, dependiendo de la viabilidad de las actividades conforme al contexto en el que se encuentre, y así permita atender "las dificultades y obstáculos del aprendizaje, así como potenciar las fortalezas de los alumnos, lo cual mejora la calidad de su práctica pedagógica" (SEP, 2017, p. 127). 
En torno a la evaluación que ocurre en la asignatura de Lengua Extranjera Inglés, tiene como finalidad central "proporcionar información sobre el grado de avance que cada alumno obtiene en las diferentes etapas del proceso de aprendizaje, para permitir al docente identificar su progreso en el dominio y competencia de inglés al término de un periodo, y valorar si las situaciones didácticas, la organización del trabajo en el aula, el uso de los materiales y el tipo de ayuda o guía proporcionada se dirigen al logro de los propósitos planteados en cada grado" (SEP, 2017, p. 177). De igual manera, lo anterior incide en las formas de interacción entre los alumnos y el docente dentro del salón de clases (SEP, 2017). Sobre la evaluación dentro del aula de idiomas, Cohen señala que debería haber una distinción entre solamente marcar reactivos con correcto o incorrecto, e ir más allá integrando el proceso evaluativo a lo largo de todo el proceso de enseñanza aprendizaje que se da dentro de este tipo de aulas en particular (2001 en Celce-Murcia, 2001).

De acuerdo al plan de estudios al docente le corresponde seleccionar el instrumento adecuado para facilitar el proceso de valoración del aprendizaje de los estudiantes y que a su vez, este sea un "proceso continuo, permanente y formativo, y no asumirla como un evento aislado que ocurre al final de un periodo determinado" (SEP, 2017, p. 177), recordando que la evaluación se centra en la comparación del desempeño del estudiante al inicio y al final del ciclo escolar de modo que se monitoree su progreso referente al desarrollo de sus habilidades comunicativas (SEP, 2017).

Pimienta (2008) considera indispensable puntualizar 4 rubros del proceso de evaluación, comprendidos en:
1. ¿Qué evaluar?
2. ¿Para qué evaluar?
3. ¿Quién evalúa?
4. ¿Cómo evalúa?

Primeramente, se pretende la evaluación de los aprendizajes y competencias propuestas en los planes y programas de estudio pertenecientes.

Su principal objetivo es contribuir a la mejora de los procesos de aprendizaje y enseñanza, este último mediante el análisis de los datos de modo que enriquezca la práctica docente.

Entre actores encargados de emitir juicios sobre los aprendizajes y competencias adquiridas se incluyen los maestros, estudiantes; siendo las autoridades educativas o padres de familia quienes asumen un valor al desempeño de la práctica docente. Inclusive los estudiantes pueden ser partícipes en este último tipo de evaluación ya que como expone Pimienta (2008) esta "no es tarea exclusiva de quien desempeña un papel particular, ni debe ser un instrumento para dominar o ejercer el poder sobre otros, sino un instrumento para tenerlo sobre nosotros y mejorar lo que desarrollamos dentro de nuestros salones" (p.29).

Durante el proceso evaluativo, se utilizan técnicas e instrumentos que son clave para recabar dicha información, los cuales deben ser planeados previamente de modo que se evite la improvisación. Este proceso debe promover la medición precisa de los datos que se pretenden evaluar evadiendo la ambigüedad y establecer fiabilidad sobre el contenido de los instrumentos.

\section{DEFINICIÓN Y TIPOS DE EVALUACIÓN}

La evaluación es un proceso que permite la toma de decisiones. En particular, para los docentes, este proceso les permite decidir en relación con el progreso de los alumnos a su cargo. Es siempre importante no confundirse con puramente calificar o medir, ya que existen aspectos cognoscitivos, afectivos y psicomotrices en el alumno que los docentes siempre tendrán que considerar (López Torres, 1999). Con lo que respecta a esta idea de evaluar como sinónimo de "medir" y "calificar", Pimienta (2008) tampoco los considera como tal, sino como procesos totalmente independientes y distintos. La calificación es descrita por Pimienta (2008) como designación de algún número, letra o palabra que exprese el desempeño del estudiante, mientras que la medición se enfoca en valoración tanto cualitativa como cuantitativa a través del diseño, análisis e interpretación de instrumentos. No obstante, él mismo determina que evaluar es "enjuiciar mediante un proceso sistemático de recopilación de los datos y la comparación con unos criterios (o normas) claramente establecidas para facilitar la toma de decisiones" (p. 33).

Es imprescindible considerar el principal objetivo de la evaluación como la mejora del "desempeño de los estudiantes e identificar sus áreas de oportunidad a la vez que es un factor que impulsa la transformación pedagógica, el seguimiento de los aprendizajes y la metacognición" (SEP, 2017, p. 125). Esta, a su vez, es encauzada en la planeación didáctica con el propósito de valorar y reflexionar sobre la práctica docente y el resultado de las habilidades desarrolladas por los estudiantes conforme al aprendizaje del inglés, así como las áreas de oportunidad que pueden darse.

La evaluación en segundas lenguas se realiza con el propósito de conocer el progreso o especificar el nivel de dominio del idioma por parte de los sujetos (Bordón, 2015). A su vez se manifiesta en diferentes etapas del proceso: al inicio, durante el desarrollo del proceso y al final de este; dando paso a lo que Pimienta (2008) entiende por evaluación diagnóstica, formativa y sumativa.

La evaluación diagnóstica corresponde a la valoración que se lleva a cabo con frecuencia al inicio del ciclo escolar o inicio de cualquier tema por lo que se requiere cualquier tipo de 
información que permita conocer los antecedentes del estudiante. Su efecto recae en la toma de "decisiones sobre la orientación del proceso al inicio del ciclo escolar y [...] recopilar información que ayudaría a explicar mucho de lo que ha sucedido con el aprendizaje de los estudiantes" (p. 38).

La evaluación formativa, tiene como función evaluar durante el desarrollo del proceso, se consolida como parte de la valoración de las habilidades desarrolladas en un punto específico del trayecto. Mientras que la evaluación sumativa, siendo esta el juicio, es planteada como una valoración del resultado del aprendizaje de los estudiantes al final del proceso, teniendo como su principal objetivo corroborar cuando el estudiante ha aprendido dependiendo de su edad y nivel educativo (Pimienta, 2008).

En contraste con Pimienta, Ur (1991) clasifica la evaluación en dos vertientes: formativa y sumativa; percibiéndolas como la retroalimentación proporcionada por los profesores. El propósito de la evaluación formativa es mejorar el proceso de enseñanza y aprendizaje de un determinado tiempo, mientras que la evaluación sumativa comprende la valoración de la recopilación de aprendizajes al final del proceso, de modo que dé a conocer la evolución del desempeño del estudiante.

En la obra Aprendizajes Clave (SEP, 2017), se menciona que las evaluaciones, además de ser diagnóstica, sumativa y formativa deben abonarse tres actores partícipes del proceso valorativo. Pimienta (2008) clasifica en tres tipos de evaluación en referencia a los participantes involucrados en este proceso: heteroevaluación, autoevaluación y coevaluación.

La primera presentada es la heteroevaluación, distingue al tipo de valoración que hace una persona sobre otra. La forma más común es el docente evaluando a cada uno de sus alumnos, también se da a la inversa, es decir el alumno evaluar al docente, o involucrar a otros agentes del sistema educativo evaluando a otros dentro de este mismo colectivo. En el proceso de enseñanza-aprendizaje, este tipo de evaluación, requiere de sistematización y constancia para poder ofrecer datos significativos que permitan mejorar la calidad del proceso anteriormente mencionado (López et al., 2012).

La segunda se especifica como una autovaloración la cual promueve la reflexión sobre el propio aprendizaje adquirido y es base fundamental del perfil de egreso planteado en el plan y programas de estudio para la educación básica (Aprendizajes Clave, 2017), debido a que la emisión de juicios que uno crea sobre otros aspectos, como la identificación rasgos y cualidades físicas, tecnologías, reconocimiento de fenómenos naturales y medio ambiente, entre otros, está presente firmemente en la formación de los educandos. En este sentido, este tipo de evaluación es un proceso en el que el participante conoce sus fortalezas y áreas de oportunidad, permitiéndole tomar acciones para potenciar su propio aprendizaje (Torres y Torres, 2005).
Por último, la coevaluación Pimienta (2008) la presenta como una evaluación entre pares, de modo que se contribuya no solo a la mejora el aprendizaje, sino que también en las actitudes entorno a un trabajo colaborativo. Este no es un medio de evaluación único entre estudiantes, también se puede dar entre los mismos maestros, directivos, entre otros. Además de lo anterior, Torres y Torres (2005) vinculan a la coevaluación con la característica de ser un proceso recíproco, el cual debe de ser una crítica constructiva cuyo objetivo principal es enriquecer el aprendizaje a nivel grupal.

Cabe destacar, que a pesar de que la autoevaluación y la coevaluación enriquecen el proceso evaluativo, Pimienta (2008) considera que existe una inclinación significativa por la heteroevaluación. Específicamente, este tipo de la valoración es realizada por el docente al el estudiante, enfocándose en los resultados del proceso de aprendizaje, con la finalidad de contribuir y mejorar en la formación de éste. A pesar de que este medio de evaluación sea considerado por Pimienta como una manera de "ejercer control sobre los estudiantes" (2008, p. 42), el docente sigue fungiendo como mediador entre el estudiante y el logro de los aprendizajes esperados.

\section{TÉCNICAS Y FORMAS PARA EVALUAR}

Considerando que evaluar no se enfoca exclusivamente en el acto de calificar y aplicar exámenes, existen una serie de técnicas para evaluar los procesos mencionados con anterioridad, de las cuales Pimienta (2008) considera como las más utilizadas para evaluar los aprendizajes de los estudiantes: la observación, la encuesta, los exámenes y los portafolios. Por ello Harmer (2015) sugiere que haya una variedad de tipos y técnicas de evaluación, tales como pruebas diagnósticas, pruebas de progreso o logro, pruebas de competencia o dominio, y evaluación de portafolios; de tal forma que, dentro de la clase de inglés, se obtengan "evidencias de diversa índole", que permita "conocer con mayor precisión los aprendizajes y las necesidades de los estudiantes" y "que el proceso de evaluación sea justo" (p. 129). Aunado a esto, O’Malley y Pierce (1996 en Dwi \& Saputri, 2017) clasifican los tipos de evaluación en auténticos, ejemplificándose como: los portafolios, la evaluación de desempeño y la autoevaluación de los estudiantes; ya que reflejan los logros de los estudiantes en relación a su aprendizaje, actitudes y motivación durante el proceso.

Cohen presenta algunas consideraciones que deben tomar los maestros de segundas lenguas a la hora de seleccionar o diseñar sus instrumentos de evaluación. Entre estos aspectos a considerar están el tener noción de lo que se va a evaluar, así como lo que se va a representar en el instrumento, además de que deben tener la mente abierta a no evaluar utilizando criterios 
tradicionales sino tener siempre alternativas de evaluación (2001 en Celce-Murcia, 2001).

Pimienta (2008) establece a los diarios de trabajo, las guías de observación estructuradas y no estructuradas, las escalas estimativas y las listas de cotejo tradicionales, los cuestionarios, las guías para evaluar los portafolios, y los exámenes, como instrumentos de valoración del conocimiento. Concerniente a la evaluación del inglés como lengua extranjera, los exámenes (exclusivamente) son clasificados por Harmer (2007) en dos categorías con base a su tipo de ítem: ítems indirectos y directos. Los ítems indirectos no miden el conocimiento general de los estudiantes acerca del idioma que se pretende aprender, agrupan a las preguntas de elección múltiple, llenar espacios, transformación y parafraseo, re-ordenar partes de oraciones. Mientras que los ítems directos se aferran a replicar interacciones de la vida cotidiana, manteniendo un grado adecuado de cultura general para el examinante.

\section{METODOLOGÍA}

Como un primer acercamiento a una comprensión sobre los procesos de evaluación de los aprendizajes en la asignatura de inglés, se alude a una investigación de tipo exploratoria, la cual con base a su enfoque cualitativo brinda perspectivas docentes conforme a la modalidad de trabajo a distancia, derivando en los siguientes objetivos:

1. Conocer la percepción de los maestros de inglés del proceso de evaluación en educación secundaria en cuanto a definiciones, técnicas, formas y criterios.

2. Detectar un primer acercamiento al proceso evaluativo durante el confinamiento por el COVID-19.

La investigación se llevó a cabo con ocho profesores de tiempo completo de la asignatura de lengua extranjera: inglés, de cuatro escuelas secundarias públicas del norte de la ciudad de Hermosillo, Sonora. La información se recolectó durante el periodo de confinamiento por la Covid-19.

El instrumento seleccionado para guiar la entrevista estructurada fue un cuestionario con preguntas abiertas. Las preguntas abiertas permiten que el interrogado emita su propia respuesta al realizarle un cuestionamiento (Babbie, 2000) sobre el tema de interés. Gento (2004) señala que "las preguntas de tipo abierto se utilizan para conocer opiniones o actitudes sobre determinados aspectos" (p. 80). En el caso del presente estudio, se formularon 12 preguntas que sirvieron de guía al entrevistador. Los cuestionamientos buscaron recopilar información sobre definiciones y ejemplificaciones por parte de los mismos sujetos sobre evaluación en relación con: definiciones, técnicas, formas y criterios, así como sobre los cambios sobre el proceso de evaluación durante la contingencia por Covid-19.
Como se mencionó anteriormente, la presente investigación siguió un enfoque cualitativo y fue de tipo exploratoria. Una investigación exploratoria busca tener un acercamiento a las definiciones, técnicas, formas y criterios para evaluar inglés en las secundarias públicas. Todo esto a través de la sistematización de la información recolectada con entrevistas semiestructuradas ya que permiten generar nuevas preguntas posibilitando profundizar en la réplica de los maestros de inglés que laboran en secundarias públicas, en caso de que la información recabada a partir de las preguntas previamente planteadas no sea suficiente

El enfoque es cualitativo, Hernández-Sampieri y Mendoza (2018) establecen que en este enfoque el investigador estudia los fenómenos de manera sistemática, pero no comienza a partir de una teoría, sino que empieza el proceso a través de la examinación de los hechos y revisando la teoría de manera simultánea. Aunado a esto, Taylor y Bogdan (1987) definen que los datos obtenidos con este tipo de enfoque son descriptivos, ya que las palabras de las personas que realizan entrevistas habladas o escritas plasman sus puntos de vista desde un espacio autónomo y se tiene una conducta observable. Un modelo de investigación que sigue este enfoque es flexible, ya que, al contrario de la metodología de recolección de datos numéricos, aquí lo que se recolecta es la opinión abierta de otras personas. En el caso de esta investigación las entrevistas se realizaron a través de videollamadas durante el periodo de receso escolar de los profesores de secundaria. Las entrevistas fueron videograbadas para facilitar la transcripción de lo emitido por los sujetos entrevistados. Posteriormente, se registró la frecuencia de las respuestas más relevantes en tablas de recurrencia con los testimonios más relevantes. La organización en tablas permitió mostrar los hallazgos cualitativos de mayor a menor recurrencia.

Blasco y Pérez (2007) mencionan que en las investigaciones con este tipo de enfoque se retratan los contextos reales, además del punto de vista e interpretaciones de las personas involucradas. La utilización de instrumentos para recoger información como las entrevistas son importantes para resultados favorables ya que es la expresión libre y sin limitaciones de los participantes. En el caso de la presente investigación los sujetos y el contexto incluyen maestros de inglés en secundarias públicas, quienes fueron seleccionados a través del muestreo por conveniencia con selección de sujetos clave.

La investigación es de tipo exploratoria. Rojas (2013) afirma que los estudios exploratorios o también llamados estudios de acercamiento a la realidad social se llevan a cabo cuando se busca reconocer, ubicar y definir problemas, con lo cual se obtendrá información que permitirá formular las bases de esquemas investigativos más definidos. De la misma manera, Babbie (2000) sostiene que los estudios exploratorios se pueden 
realizar cuando el interés sobre el tema es relativamente nuevo, o cuando hay fenómenos persistentes. En este segundo caso los resultados del estudio exploratorio, permiten vislumbrar resultados, que pueden ser utilizados posteriormente en estudios más extensos.

La técnica seguida para recolectar información fue la entrevista semiestructurada que se ubica dentro de las técnicas basadas en la interrogación oral descritas por Gento (2004) se encuentra la entrevista. De acuerdo a Gento (2004) "la entrevista trata de obtener información sobre un determinado objeto de estudio o investigación, de forma que una persona o personas entrevistadas responden directamente al propio entrevistador" (p. 63). El tipo de entrevista realizada para recolectar información en esta investigación fue semiestructurada, por lo que las preguntas a realizarse fueron previamente diseñadas en una guía que podía ser modificada. Debido a la naturaleza de la entrevista semiestructurada, la función del entrevistador en esta situación es preparar y formular las preguntas conforme se va desarrollando la entrevista, siendo respetuoso con el entrevistado (Gento, 2004).

Taylor y Bogdan (1987) definen la entrevista a grandes rasgos como el encuentro entre entrevistador y entrevistado. Es en este encuentro, en donde se busca llegar a conocer información referente a situaciones de interés para el entrevistador, las cuales son expresadas con las palabras y percepción del entrevistado, en este caso los maestros de inglés seleccionados como muestra.

Una vez recolectada la información se analizaron las respuestas de cada uno de los participantes seleccionados como muestra.

\section{RESULTADOS Y DISCUSIÓN}

\section{Categoría: Definiciones}

\begin{tabular}{|l|c|}
\hline Respuestas & Recurrencia \\
\hline $\begin{array}{l}\text { Reconocimiento del progreso de los } \\
\text { estudiantes y futuras mejoras. }\end{array}$ & 6 \\
\hline Proceso constante & 4 \\
\hline Retroalimentación para el docente & 2 \\
\hline Es un requisito impuesto por la institución & 1 \\
\hline
\end{tabular}

\section{Tabla 1. Reconocimiento del progreso de los estudiantes y} futuras mejoras. ¿Cómo es definida la evaluación?

La evaluación es percibida principalmente, entre los profesores de secundaria, como la valoración del aprendizaje de los estudiantes, de modo que hay un reconocimiento de la adquisición de conocimientos para futuras intervenciones como volver a enseñar ciertos temas cuyos resultados no fueron favorables. Lo anterior también funge como una retroalimentación de la misma práctica docente, con la finalidad de identificar y mejorar el desempeño de los estudiantes (SEP, 2017).
Cabe destacar que la frecuencia con la que se acuerda el proceso evaluativo es constante; no obstante, para ciertas instituciones de educación básica, este proceso incluye como requisito un examen escrito el cual es imprescindible. Concluyendo así que los maestros, en concordancia con Pimienta (2008), no consideran la evaluación exclusiva al acto de calificar.

\section{Pre-covid}

\begin{tabular}{|l|c|}
\hline Respuestas & Frecuencia \\
\hline $\begin{array}{l}\text { Revisión de actividades de clase en } \\
\text { cuaderno de trabajo o libro de texto }\end{array}$ & 6 \\
\hline $\begin{array}{l}\text { Conceder prioridad a la } \\
\text { participación }\end{array}$ & 5 \\
\hline $\begin{array}{l}\text { Implementación de proyectos } \\
\text { como producto final (discurso, } \\
\text { diálogo, presentaciones) }\end{array}$ & 4 \\
\hline Cuaderno de trabajo & 6 \\
\hline Exámenes & 6 \\
\hline
\end{tabular}

Tabla 2. Estrategias, técnicas e instrumentos de evaluación utilizadas por el docente

Harmer (2015) establece la existencia de una gran variedad de instrumentos, técnicas y estrategias de evaluación, sin centrarse en una en específico, lo cual conlleva a que los resultados dados por los docentes en cuanto a las estrategias y técnicas de evaluación sobresaliente entre la comunidad sean las actividades realizadas en clase. Esta variedad no centrada en evaluaciones estandarizadas permite conocer con mayor precisión el progreso de los estudiantes y la efectividad de la práctica docente (Harmer, 2015). Los resultados previamente expuestos sitúan a los cuadernos de trabajo y libros de texto como instrumentos indispensables para la valoración de los aprendizajes. A la par con los exámenes, este último es respaldado por Harmer (2015) y Pimienta (2008) como instrumentos indispensables en la evaluación de los aprendizajes.

\begin{tabular}{|l|c|}
\hline Respuestas & Recurrencia \\
\hline Trabajo en clase & 6 \\
\hline Actitud y disciplina & 4 \\
\hline Exámenes & 4 \\
\hline $\begin{array}{l}\text { Contribución a la adquisición y } \\
\text { fortalecimiento de conocimientos y } \\
\text { la producción del mismo }\end{array}$ & 4 \\
\hline $\begin{array}{l}\text { Cuidado de los sentimientos, } \\
\text { actitudes y motivación del } \\
\text { estudiante }\end{array}$ & 3 \\
\hline
\end{tabular}

Tabla 3. Criterios y consideraciones de aspectos evaluativos en una clase de inglés como lengua extranjera 
Conforme a las explicaciones dadas por los docentes, la implementación de exámenes se vuelve un aspecto evaluativo obligatorio por parte de la administración del plantel y de las autoridades educativas encargadas. Sin embargo, se le asigna mayor porcentaje al trabajo en clase, así como a la actitud, disciplina y asistencia de los alumnos a las sesiones programadas. Hay que destacar que estos tres rubros mencionados con anterioridad no son considerados por los docentes entrevistados con el propósito de penalizar a los estudiantes en su calificación final, sino para apoyarlos.

Dentro de la evaluación entran en juego elementos intangibles o subjetivos como los sentimientos, actitudes y valores del alumno. Es decir, importa cómo afecta la calificación para que sigan esforzándose en la clase de inglés; hay maestros que mencionaron que más que aprender un idioma también es importante motivar a los aprendices. Y a pesar de que a veces no se quiera evaluar con un instrumento riguroso como un examen se debe realizar porque así lo impone la escuela. Como es presentado por Pimienta (2008) la evaluación es un proceso tanto cuantitativo, como cualitativo en la formación de aprendizajes.

\begin{tabular}{|l|c|}
\hline Respuestas & Recurrencia \\
\hline Diariamente & 8 \\
\hline Trimestral & 4 \\
\hline Al final de cada unidad & 2 \\
\hline
\end{tabular}

Tabla 4. Momentos de evaluación en una clase de inglés como lengua extranjera

Los momentos para evaluar varían dependiendo de los objetivos y parámetros que establezca cada docente y la institución, pero existe coincidencia en la evaluación diaria, que como ya se ha mencionado esta se lleva a cabo a través del registro de la participación, actitud y trabajos en clase, reduciéndose en evaluación formativa y sumativa. Es formativa porque permite a los docentes darse cuenta de las habilidades desarrolladas por sus estudiantes (Pimienta, 2008), dichas habilidades no únicamente se limitan al aprendizaje del inglés, sino también al sentido formativo de valores para la vida como ciudadano fuera del contexto escolar que busca el perfil de egreso de la educación obligatoria (SEP, 2018). La evaluación sumativa se manifiesta en valorar numéricamente al final de ciertos periodos, en el caso de las clases de lengua extranjera: inglés en secundaria, al finalizar cada uno de los tres trimestres del ciclo escolar. En este sentido la mayoría de los docentes consideraron que la evaluación de sus alumnos, no está limitada a que éstos logren los aprendizajes esperados, sino que también se extiende a aspectos cualitativos observados durante el curso.

\section{Durante covid}

\begin{tabular}{|l|c|}
\hline Respuestas & Recurrencia \\
\hline Reducción de actividades & 5 \\
\hline
\end{tabular}

\begin{tabular}{|l|l|}
\hline Evaluación de temas esenciales & 1 \\
\hline $\begin{array}{l}\text { Las instituciones solicitaban aprobar a los } \\
\text { alumnos }\end{array}$ & 7 \\
\hline Cambio en los criterios de evaluación & 1 \\
\hline $\begin{array}{l}\text { Abordaje de contenidos de manera } \\
\text { superficial }\end{array}$ & 1 \\
\hline $\begin{array}{l}\text { Implementación de un portafolio de } \\
\text { evidencias con fotografías de trabajos }\end{array}$ & 1 \\
\hline No se aplicó examen & 1 \\
\hline Evitar análisis en la revisión de tareas & 1 \\
\hline $\begin{array}{l}\text { Uso de las redes sociales como medio de } \\
\text { comunicación y recepción de tareas }\end{array}$ & \\
\hline
\end{tabular}

\section{Tabla 5. Adecuaciones curriculares por COVID-19}

En la tabla 3, se manifestó la solicitud de no asignar una calificación reprobatoria (5) a los estudiantes que no muestran desempeño satisfactorio, debido a criterios proporcionados por la SEP durante los meses de confinamiento en el ciclo 20192020. Por lo tanto, el proceso de la evaluación sumativa presentó una alteración al no aplicar exámenes, reducir el número de actividades y los temas a evaluar. De igual manera, el extenso número de estudiantes matriculados por aula fue una razón para eludir profundizar en la revisión de las tareas recibidas y en el contenido de las unidades planteadas por los libros de texto.

El confinamiento por el COVID-19 forzó la educación a distancia, tomando como medio de comunicación y recepción de tareas el uso de redes sociales, ocasionando que los criterios de evaluación establecidos al inicio de ciclo escolar, en especial los que contaban con mayor ponderación eran los elementos intangibles como la actitud y disciplina, los cuales han perdido su relevancia al trabajar a distancia a causa de la complejidad que ocasiona su valoración a través de un dispositivo electrónico.

La percepción de los maestros de inglés del proceso de evaluación en educación secundaria tiende a ser semejante a pesar de encontrarse en diferentes zonas escolares de la ciudad.

La idea que se tiene en cuanto a definiciones, tiende a ser similar entre sí refiriéndose a la evaluación como el reconocimiento del progreso de los estudiantes a lo largo de un periodo de tiempo específico (delimitado diariamente), con el objetivo de modificar la propia práctica docente y las estrategias de enseñanza para beneficio del aprendizaje de los estudiantes.

Es peculiar el hecho de que la evaluación no se relaciona en ningún momento con el término de calificar, tal y como afirman algunos autores, sino que se basan en los modos de trabajo más que asignar un valor numérico a este proceso.

Conforme a la reflexión docente, se evalúa con el propósito de contribuir a la adquisición y fortalecimiento de conocimientos 
y la producción del mismo, excluyendo los exámenes y coincidiendo en la utilidad del cuaderno de trabajo como instrumento, técnica y criterio fundamental para valorar los aprendizajes, dándole relevancia a las actitudes y disciplina como juicio de evaluación. Esto último con la finalidad de cuidar los sentimientos y motivación del estudiante.

En relación al segundo objetivo, el proceso evaluativo durante el confinamiento por el COVID-19, implementó inesperadamente el trabajo a distancia en todos los niveles educativos en México. Y aunque este tipo de labor sea percibido como un reto por todos los actores involucrados en el quehacer educativo, la educación a distancia se conserva y ha trascendido desde el siglo XIX en Estados Unidos. En este tiempo los estudiantes eran evaluados a través de los exámenes y trabajos que enviaban por correo a sus profesores, progresando a su vez a las clases por medio de la radio y la televisión en el siglo XX, modificando así los métodos de valoración de los aprendizajes (Rangel, 2020). No obstante, las circunstancias inesperadas, llevó a que el sistema educativo y su vez los docentes no estuvieran capacitados causando que los criterios de evaluación fueran modificados, excluyendo exámenes y los elementos intangibles, dejando solo los trabajos hechos en casa como un factor indispensable. Esto, aunado a la aprobación obligatoria del estudiantado por parte de las instituciones, les dio la oportunidad de acreditar el ciclo escolar con el mínimo esfuerzo.

\section{CONCLUSIÓN}

En conclusión, a los hallazgos descubiertos a través de las entrevistas a docentes, se deduce que la práctica del examen va fungiendo como un instrumento de evaluación menos constante, reduciendo sus técnicas, formas y criterios exclusivamente a la evaluación diaria del trabajo en clase. Esto fue previo a la contingencia sanitaria. Durante el confinamiento, la implementación y desarrollo de la escuela a distancia provocó una alteración en las técnicas, formas y criterios de evaluación; lo cual resulta en la evaluación únicamente de los trabajos recibidos, complicando la valoración única del modelo de clase presencial que se estaba trabajando: disciplina y asistencia.

Para finalizar, es necesario destacar la inexistente diferenciación entre técnicas, formas y criterios por parte de los profesores, ya que la similitud de sus percepciones dentro de estos tres rubros es evidente en la tabla 1 y 2 , donde la evaluación es percibida como reconocimiento del progreso de los estudiantes y futuras mejoras, evidenciando la falta de enfasís en cuanto a las estrategias, técnicas e instrumentos de evaluación utilizadas por el docente, ya que las respuestas eran generalizadas a la revisión de actividades de clase en cuaderno de trabajo o libro de texto y no clasificadas en alguna de las categorías expuestas con anterioridad. Si bien la concepción inmediata de la evaluación podría dirigirse a la medición del conocimiento mediante la aplicación de exámenes, su definición y la capacidad de clasificarlos en alguna característica que conlleva la evaluación concedidos por los docentes entrevistados es carente.

En concordancia con Díaz y Hernández (2000, en Córdoba, 2006, p.1), "la evaluación del proceso de aprendizaje y enseñanza es una tarea necesaria, en tanto que aporta al profesor un mecanismo de autocontrol que la regula y le permite conocer las causas de los problemas u obstáculos que se suscitan y la perturban". A su vez los profesores perciben la evaluación como una oportunidad de mejora de su misma práctica docente, analizando los resultados de las evaluaciones de modo que se conozca el conocimiento actual del estudiante y el avance (en su caso) de su aprendizaje a lo largo del ciclo escolar, para tomarse como punto de partida hacia el perfeccionamiento del proceso de enseñanza abordando las áreas de oportunidad que limitan el máximo potencial de los estudiantes.

\section{REFERENCIAS}

[1] Babbie, E. (2000). Fundamentos de la investigación social. International Thomson Editores.

[2] Blasco, J. E. y Pérez, J. A. (2007). Metodologías de investigación en las ciencias de la actividad física y el deporte: ampliando horizontes. Editorial Club Universitario.

[3] Bordón, T. (2015). La evaluación de segundas lenguas (L2). Balance y perspectivas. Revista Internacional de Lenguas Extranjeras, 4, 9-3. doi: 10.17345/rile20159-30

[4] Cohen, A. (2001). Second Language Assessment. En M. Celce-Murcia (Ed.), Teaching English as a Second or Foreign Language $\left(3^{\text {rd }}\right.$ ed.) (pp. 515-534). National Geographic Learning.

[5] Córdoba Gómez, F. J. (2006). La evaluación de los estudiantes: una discusión abierta. Revista Iberoamericana De Educación, 39(7), 1-8. doi:10.35362/RIE3972537

[6] Dwi, R. \& Saputri, L. (2017). The authentic assessment to measure students' English productive skills based on 2013 Curriculum. Indonesian Journal of Applied Linguistics. 7(2), 263-273. doi: 10.17509/ijal.v7i2.8128.

[7] Education First. (2019). Índice de Competencia en Inglés. Education First. Obtenido de: https://www.ef.com.es/epi/regions/latinamerica/mexico/

[8] Gento, S. (2004). Guía práctica para la investigación en educación. Sanz y Torres.

[9] Gultom, E. (12 de mayo, 2016). Assessment and Evaluation in EFL Teaching and Learning. Proceedings of International Seminar on English. Language and Teaching. 4(1), 190-198.

[10] Harmer, J. (2007). The Practice of English Language Teaching. Pearson Longman.

[11] Harmer J. (2015). The Practice of English Language Teaching. Pearson Education Limited.

[12] Hernández-Sampieri, R. y Mendoza C. (2018). Metodología de la investigación. Las rutas cuantitativa, cualitativa y mixta. Mc Graw Hill Education.

[13] Organización de las Naciones Unidas para la Educación, la Ciencia y la Cultura. (2020). Respuesta del ámbito educativo a la COVID-19: 
Preparación para la reapertura de las escuelas: documento de trabajo. UNESCO. Obtenido de: https://unesdoc.unesco.org/ark:/ 48223/pf0000373401_spa?posInSet=97\&queryId=N -a01e63fe-9128-4751-b60c-c0cd6fd50218\&fbclid=I wAR18HMPIUZIdfoctdLYX_nTD7a3nnQaCDq91J zPr6maUzWD_7FTutWNKIU

[14] López, R., Gutiérrez, M., Vázquez, S., Benet, M., Tereñes, C. y Legrá, S. (2012). La evaluación en la educación a distancia en la búsqueda de un aprendizaje desarrollador. MediSur, 10(2),102-108. Obtenido de:

https://www.redalyc.org/articulo.oa?id=1800/180023438016

[15] López Torres, M. (1999). Evaluación educativa. Trillas.

[16] Mexicanos Primero. (2015). Sorry. El aprendizaje del inglés en México. Mexicanos Primero. Recuperado de: https://www.cce.org.mx/wp-content/uploads/2014/07/Sorry-ElAprendisaje-del-Ingles-en-Mexico.pdf

[17] Pimienta, J. (2008). Evaluación de los aprendizajes. Un enfoque basado en competencias. Pearson Educación.

[18] Rangel, R. (2020). La Era del Aprendizaje Activo. [formato de la clase, classroom, google docs]. Obtenido de: https://classroom.google.com/u/0/c/MTIzMDE4NDU2Njcy

[19] Rojas, R. (2013). Guía para realizar investigaciones sociales. Plaza y Valdés Editores.

[20] Secretaría de Educación Pública. (2017). Aprendizajes clave para la educación integral. SEP.

[21] Secretaría de Educación Pública. (2020). Principales Cifras del Sistema Educativo Nacional 2019-2020. SEP. Recuperado de: https://www.planeacion.sep.gob.mx/Doc/estadistica_e_indicadores/princip ales_cifras/principales_cifras_2019_2020_bolsillo.pdf

[22] Taylor, S. y Bogdan, R. (1987). Introducción a los métodos cualitativos de investigación. Paidós.

[23] Tomlinson, B. (2005). Testing to learn: a personal view of language testing. ELT Journal, Oxford University Press, 59 (1), 49-46. doi: 10.1093/elt/cci005

[24] Torres, M. E. y Torres, C. M. (2005). Formas de participación en la evaluación. Educere, 9(31), 487-496. Obtenido de: https://www.redalyc.org/articulo.oa?id=35603109

[25] Ur, P. (1991). A course in language teaching. Practice and Theory. Cambridge University Press. 